\title{
Separation of Heavy Rare-Earth Elements from Light Rare-Earth Elements Via Solvent Extraction from a Neodymium Magnet Leachate and the Effects of Diluents
}

\author{
Marino Gergoric $^{1}$ (D) $\cdot$ Christian Ekberg $^{1} \cdot$ Britt-Marie Steenari $^{1} \cdot$ Teodora $^{\text {Retegan }}{ }^{1}$
}

Published online: 2 February 2017

(c) The Author(s) 2017. This article is published with open access at Springerlink.com

\begin{abstract}
In recent decades, rare-earth elements (REEs) have seen a considerable increase in usage in modern technologies and the so-called green energy sources. The REEs are currently regarded to be among the most critical elements by the European Union (EU) and the United States (USA). Large investments are made in the research of recycling of the REEs from end-of-life products and E-scrap. One potential source for recycling of larger amounts of neodymium and dysprosium are end-of-life neodymium magnets. In this work, the selective extraction of REEs from a sulfuric media leachate (containing $\mathrm{Nd}$, Dy, Pr, Gd, Co, and B) obtained by selective roasting of $\mathrm{NdFeB}$ waste and leaching was investigated. The extracting agent D2EHPA (di-(2-ethylhexyl) phosphoric acid) diluted in Solvent 70, hexane, octane, cyclohexanone, chloroform, 1-octanol, and toluene was used for the investigation of the effects of using different diluents on the extraction of REEs and the separation between the light and the heavy REEs. The concentrations of D2EHPA in the used diluents were 0.3, 0.6, 0.9, and 1.2 M. The highest separation factors between the heavy and the light REEs were achieved using 0.3 M D2EHPA in hexane, while no B or Co extraction was measurable. The REEs were completely extracted as a group using $0.9 \mathrm{M}$ or $1.2 \mathrm{M}$ D2EHPA in either octane or hexane, also with no B or Co extraction. The aliphatic nonpolar diluents showed better properties than the aromatic and polar ones. The complete
\end{abstract}

The contributing editor for this article was D. Panias.

Marino Gergoric

marino@chalmers.se

1 Nuclear Chemistry and Industrial Materials Recycling, Chemistry and Chemical Engineering, Chalmers University of Technology, 41296 Gothenburg, Sweden stripping of REEs from the loaded organic phases was proven to be efficient using hydrochloric acid at concentrations of $2 \mathrm{M}$ or higher.

Keywords Neodymium magnets $\cdot$ Recycling $\cdot$ Solvent extraction - D2EHPA - Diluents · Heavy and light rareearth elements

\section{Introduction}

Rare-earth elements (REEs) are a group of 17 elements, consisting of the 15 lanthanides plus scandium and yttrium. REEs are used in a wide range of products, such as fluorescent lamps, magnets, superconductors, lasers, ceramics, semiconductors, catalysts, and thermal neutron absorbents [1]. Many of these applications are important for the development of environmentally friendly technologies for transport, lighting, energy storage, and the manufacturing of chemicals. The demand for REEs is therefore increasing. Since they are mined in only a few countries and their prices have varied in recent years, their availability is considered critical by both the EU and the USA [2]. The REEs are considered the most critical based on their economic importance and supply risk, as stated in the EU report on critical elements from 2013 [3].

Due to the uncertainty in the supply of virgin REEs, the possibilities of recovering and reusing them from spent products are presently being investigated [2, 4-6]. Some products are rich in materials containing significant amounts of REEs. One such material is neodymium-ironboron $(\mathrm{NdFeB})$ magnet scrap, which could be a source of neodymium and dysprosium, added to increase the Curie temperature of the magnet, and some other REEs, depending on the type of magnet. The material is 
essentially an iron alloy, meaning that two-thirds of the material is made up of iron, which poses the greatest challenge in further recycling, since iron is not currently a critical metal and is rather cheap [7]. Apart from the REEs and iron, the most common element present in the magnets is cobalt [8]. Cobalt itself is a valuable element and is worth recycling [9].

Recycling of discarded magnets requires removal of the magnet from the device where it has been in use, demagnetization, size reduction, and a hydrometallurgical (leaching and separation from the liquid phase) or pyrometallurgical (high-temperature processes and separation in molten salts) separation of the constituent elements $[2,6,10]$.

Solvent extraction, or liquid-liquid extraction, is a commonly used hydrometallurgical method for the recovery and removal of metal ions from aqueous solutions [10]. The aqueous phase, i.e., the leachate obtained by acid dissolution of the waste, in this case containing dissolved REEs along with cobalt and other contaminating metals, is mixed with an organic phase containing ligand molecules ("extractants") designed to transfer selectively the chosen ions to the organic phase. The combination of solvent and ligand can be varied in order to optimize the properties of the organic phase for a certain separation process. In some cases, a third component, called a modifier, can be added in order to modify an important property of the organic phase [11]. However, it is difficult to separate the REEs from each other due to their chemical similarity $[2,10]$.

The separation of groups of related REEs from other groups of REEs has been shown to be technically more feasible, and this approach was chosen in the present work. Di-(2-ethylhexyl) phosphoric acid (D2EHPA) has shown good versatility as an extractant for the separation of REEs due to its chemical stability, good kinetics in extraction, good loading and stripping properties, and availability in commercial quantities [12]. Therefore, D2EHPA was chosen as the extractant in this work. The aim of the present work was to study the effects of modifications in the composition of the organic phase in the separation of light REEs (LREEs) from heavy REEs (HREEs) resulting from sulfuric acid leaching of end-of-life neodymium magnets.

\section{Background}

The recovery and recycling of REEs and other metals from magnets can be performed by hydrometallurgical methods. This approach includes leaching of these elements with mineral acids followed by solvent extraction for isolation and separation of the metals of interest [6].

In 2013, Lee et al. [13] reported the leaching of $\mathrm{NdFeB}$ magnet scrap with $\mathrm{HCl}, \mathrm{HNO}_{3}, \mathrm{H}_{2} \mathrm{SO}_{4}$, or $\mathrm{NaOH}$. The processes were optimized with regard to temperature, leaching time, concentration of leaching reagent, and solidto-liquid ratios. $\mathrm{HCl}$ and $\mathrm{H}_{2} \mathrm{SO}_{4}$ showed the best performance. Neodymium was successfully recovered from an optimized $\mathrm{H}_{2} \mathrm{SO}_{4}$ leaching solution with $75.41 \%$ yield by precipitation. The optimal conditions were a solid-to-liquid ratio (S:L) of $20 \mathrm{~g} / \mathrm{L}, 15 \mathrm{~min}$ leaching time, and $3 \mathrm{M}$ hydrochloric acid or 1.5 M sulphuric acid. In 2014, Yoon et al. [14] carried out a similar investigation and reported that increasing the leaching temperature gave an increased leaching effect of $\mathrm{H}_{2} \mathrm{SO}_{4}$ when leaching the $\mathrm{NdFeB}$ magnetic scrap. The optimal leaching conditions were determined to be $4 \mathrm{~h}$ leaching time at $70{ }^{\circ} \mathrm{C}$ using $3 \mathrm{M} \mathrm{H}_{2} \mathrm{SO}_{4}$. The main obstacle in leaching of the $\mathrm{NdFeB}$ magnet scrap is leaching the iron, which usually makes up around twothirds of the $\mathrm{NdFeB}$ magnet, into the solution. The idea of selectively precipitating the REEs and as little as possible the other constituent materials except for iron was the research focus of much interest [2]. Results obtained by Önal and co-workers [15] showed that powdered $\mathrm{NdFeB}$ magnets can be selectively leached, with iron remaining in the solid residue. The powdered samples were transformed into a sulfate mixture by mixing the powder with sulfuric acid in alumina crucibles with the acid:magnet ratios $(\mathrm{g} / \mathrm{g})$ of $2.15(12 \mathrm{M}), 3.2(13.5 \mathrm{M}), 4.3(14.5 \mathrm{M})$, and 8.6 $(16 \mathrm{M})$. The mixtures obtained were then dried in a muffle furnace at $110^{\circ} \mathrm{C}$ for $6-24 \mathrm{~h}$. The dried samples were then treated at $650-800^{\circ} \mathrm{C}$ for $15-120 \mathrm{~min}$, resulting in a selective roasting process. The obtained calcines were later leached with demineralized water for $15 \mathrm{~min}$ to $24 \mathrm{~h}$ at $225 \mathrm{rpm}$ on a shaker. This process led to $95-100 \%$ extraction efficiencies for $\mathrm{Nd}$, Pr, Dy, and Gd, while Fe remained in the residue after leaching. The leachate can be used later on in other hydrometallurgical processes and was used in this investigation as the aqueous phase in solvent extraction, as a follow-up process.

As already mentioned, REEs are challenging to separate from each other in complex mixtures and natural occurring ores. Separating the REEs can be done in various ways, such as by chemical separations, fractional crystallization, ion-exchange methods, and solvent extraction. Solvent extraction has been proven to be the best method for separating REEs from chemically complex waste streams [16], and it is the only method used on a commercial scale today. It can give materials of up to $99.99 \%$ purity [17].

The REEs are usually divided into two groups, known as the light rare-earth elements (LREEs), i.e., lanthanum through gadolinium; and the heavy rare-earth elements (HREEs), i.e., terbium through lutetium plus yttrium [18]. The common property of the LREEs is the increasing number of unpaired electrons from 0 to 7 , while the HREEs have the increasing number of paired electrons from 8 to 14. Their specific electronic configuration, where the $4 \mathrm{f}$ 
orbitals are 'hidden' behind the $4 \mathrm{~d}$ and $5 \mathrm{~d}$ orbitals, in which the REEs have the same number of electrons, results in similar characteristics of the elements in this group. One example is that the stability constants of metal ion complexes with a particular ligand show only slight differences [17]. This means that the tendency of the formed complexes to be dissolved in the organic phase in a solvent extraction will be similar for all the REEs. Separating the LREEs from the HREEs is a more feasible approach than separating the closely related REEs, since the farther away they are in a chemical period, the bigger the difference between the properties of the complexes formed are expected to be. Separating the LREEs from the HREEs could especially be of economic importance since the HREEs are considered the most critical, with their prices being significantly higher than those of the LREEs [3]. In order to achieve a good separation of the REEs, it is therefore necessary to choose a proper, and as selective as possible, complexing agent [19]. In the separation of the REEs, organophosphorous extractants are commonly used. As already mentioned, di(2-ethylhexyl)-phosphoric acid (D2EHPA), an acidic extractant, is commonly used on a larger industrial scale [20]. The selectivity order for extracting rare earths from $0.5 \mathrm{M} \mathrm{HCl}$ solution with $0.75 \mathrm{M}$ D2EHPA in toluene was found by Peppard and coworkers to be $\mathrm{Lu}>\mathrm{Yb}>\mathrm{Tm}>\mathrm{Tb}>\mathrm{Eu}>\mathrm{Pm}>\mathrm{Pr}>-$ $\mathrm{Ce}>\mathrm{La}$, with the $\log$ of the distribution coefficient increasing linearly with the atomic number of the rare earth [21].

D2EHPA has also been used to separate $\mathrm{Sm}, \mathrm{Eu}$, and Gd from the other rare earths in a mixed nitrate-chloride leachate from monazite, with the general formula $(\mathrm{Ce}, \mathrm{La}) \mathrm{PO}_{4}$ [22]. Furthermore, in many studies [23], it was shown that a typical separation factor between adjacent REEs using the D2EHPA extractant is in the region of 2.5. The separations of neodymium and dysprosium ions in solution have been achieved with organophosphorous extractants, such as PC-88A by Tanaka and co-workers [6]. A separation factor of 525 was obtained at $\mathrm{pH}$ 1.1. Scrubbing of the neodymium ions that are extracted alongside dysprosium ions is carried out with dysprosium chloride or dilute hydrochloric acid solutions [6].

In solvent extraction, the solvent, or organic phase, is made up of an extractant dissolved in a diluent. In some cases, pure extractant is used, but it is more common to use a diluent since many extractants are viscous in their undiluted form. A third component, a modifier, may be added to the organic phase to prevent the third phase formation. Chemical energy is spent in transferring the metal from the aqueous phase, and it would seem advantageous to use the extractant in a highly concentrated form, but practical problems have led to $10-40 \%$ extractant often being considered optimal [11]. Some common diluents used in solvent extraction are kerosene, $n$-hexane, $n$-octane, benzene, cyclohexanone, and toluene. The type of diluent in the organic phase plays a significant role in the formation of the complexes, and can thus affect the distribution ratios of the metals in the leachate that are of interest. The properties of the diluents can significantly affect the efficiency of extraction. The ability of the diluent to form hydrogen bonds can affect the solubility of the extractants in the organic phase. The polarity of the diluent can also significantly affect the extraction process since the solubility of the neutral complex in the organic phase is inversely proportional to the polarity of the organic diluent $[24,25]$. In this work, various diluents were applied in order to investigate the dependence of the separation factor for the individual REEs on the organic phase composition.

\section{Theory}

The distribution ratio $(D)$ is the most important parameter involved in the solvent extraction along with the separation factor $(\alpha)$. These are determined using Eqs. 1 and 2, respectively:

$D_{\mathrm{A}}=\frac{[\mathrm{A}]_{\mathrm{org}}}{[\mathrm{A}]_{\mathrm{aq}}}$,

$\alpha_{\mathrm{A} / \mathrm{B}}=\frac{D_{\mathrm{A}}}{D_{\mathrm{B}}}$,

where $[\mathrm{A}]_{\mathrm{org}}$ and $[\mathrm{A}]_{\mathrm{aq}}$ are the equilibrium concentrations of the metal of interest in all its existing species in the organic and aqueous phases, respectively. Consequently, the separation factor is the ratio between the distribution ratios of the metal 1 and 2 that are of interest. The separation factor represents the selectivity between these two metals in the extraction [26].

Many authors [26, 27] have reported that the extraction of the REEs with organophosphorous extractants (here called HR) occurs according to the reaction path described in Eq. 3:

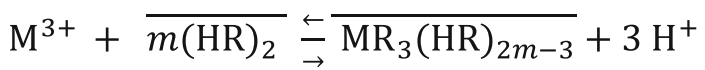

where $\mathrm{M}^{3+}$ is the lanthanide ion in the solution; and HR is the organophosphorous extractant in the organic phase occurring as a dimer $(\mathrm{HR})_{2}$ and as $\mathrm{MR}_{3}(\mathrm{HR})_{2 \mathrm{~m}-3}$ in the complex formed, soluble only in the organic phase. Following the Le Chatelier's principle, looking at Eq. 3, it is possible to conclude that a decrease in the $\mathrm{pH}$ of the leachate will consequently lead to lower distribution ratios for the metals extracted. The change in $\mathrm{pH}$ may therefore be used to steer the selectivity of extraction between the elements of interest. 


\section{Experimental}

The neodymium magnet leachate was produced by Önal et al. through sulfation, selective roasting, and water leaching [15]. The neodymium magnet leachate composition was measured using inductively coupled plasma (ICPOES) by diluting the sampled leachate with $0.5 \mathrm{M} \mathrm{HNO}_{3}$ $\left(65 \%\right.$, suprapur ${ }^{\circledR}$, Merck) and then performing the measurement. Experiments were carried out in triplicate. The $\mathrm{pH}$ value of the solution was measured using the Meter$\mathrm{Lab}^{\mathrm{TM}}$ PHM $240 \mathrm{pH} /$ ion Meter $\mathrm{pH}$ electrode. The sulfate ion concentration needed to be determined was performed by the precipitation of $\mathrm{BaSO}_{4}$ using $\mathrm{BaCl}_{2}(99.999 \%$ trace metal basis, Sigma-Aldrich) dissolved in MQ water. The obtained precipitate was filtered through a polypropylene filter paper $(0.45 \mu \mathrm{m}$, VWR), washed with $20 \mathrm{~mL}$ MQ water, and then finally dried for $48 \mathrm{~h}$ in the fume hood under normal ventilation conditions at a temperature of $25 \pm 1{ }^{\circ} \mathrm{C}$. The mass difference between the dried filter paper containing the precipitate and the mass of filter paper before filtration was used as the mass of $\mathrm{BaSO}_{4}$.

\section{Investigation of the Kinetics, the Effect of the Concentration of D2EHPA, and the Effect of Diluent on Solvent Extraction}

In the investigation of the extraction kinetics, the effect of D2EHPA concentration, and the effect of the diluent, the following organic phases were used: D2EHPA (97\%, Sigma-Aldrich) diluted in Solvent 70 (hydrocarbons C11C14, =aromatics, Statoil, Sweden), hexane (95\%, anhydrous, Sigma-Aldrich), octane (98\%, reagent grade), toluene $(99.8 \%$, anhydrous, Sigma-Aldrich), cyclohexanone ( $\geq 99 \%$, ACS reagent, Sigma-Aldrich), 1-octanol ( $\geq 99 \%$, ACS reagent, Sigma-Aldrich), and chloroform ( $\geq 99.9 \%$, containing amylenes as stabilizer, SigmaAldrich). All the organic phases were pre-equilibrated with an equal amount of MQ water. The aqueous phase used was the obtained leachate. An Ika Vibrax Vxr basic shaking machine (shaking speed of 1750 vibrations/min), equipped with an adjacent thermostatic water bath, was used for the shaking experiments. The experiments were performed in $3.5 \mathrm{~mL}$ glass vials at $25 \pm 1{ }^{\circ} \mathrm{C}$, and an aqueous-to-organic phase ratio, $\Theta=1$. All the experiments were performed in triplicates. In all the cases, the error bars on the graphs were omitted due to lack of graph clarity and insufficient scientific meaning for this specific case. The vials before each sampling were centrifuged at a rotation speed of $2000 \mathrm{rpm}$ for $1 \mathrm{~min}$. The sampled aqueous phases, as well as the aqueous phases before extraction, were diluted with $0.5 \mathrm{M} \mathrm{HNO}_{3}$ and analyzed using ICPOES. The distribution ratios were calculated as the mass balance of these measurements.
The concentration of 0.6 M D2EHPA in the aforementioned diluents was used. It was determined that the time needed for reaching the required distribution ratios for the REEs ( $\mathrm{Nd}, \mathrm{Pr}, \mathrm{Gd}$, and Dy) to establish the equilibrium between the organic and aqueous phases was less than $3 \mathrm{~min}$ in Solvent 70, hexane, octane, toluene, and 1-octanol, while in cyclohexanone and chloroform, it took $5 \mathrm{~min}$ for the equilibrium to be reached. No cobalt whatsoever was extracted under any of the conditions, which shows that REEs can be extracted selectively without any cobalt transfer into the organic phase. Boron distribution ratios reach a mere 0.1 in Solvent 70 after $5 \mathrm{~min}$, with some minor extraction in 1-octanol, but no other extraction was realized in other diluents. Further experiments were performed according to these results.

The effects of the concentration of D2EHPA and diluent on the solvent extraction of the REEs out of the leachate were investigated in order to determine the optimal organic phase composition for the solvent extraction process.

The extractant D2EHPA was used in the concentrations of $0.3,0.6,0.9$, and $1.2 \mathrm{M}$ diluted in Solvent 70, hexane, octane, toluene, cyclohexanone, 1-octanol, and chloroform. All the organic phases used in this work were pre-equilibrated with MQ water before performing the experiments.

\section{Investigation of pH Effect on Extraction}

Extraction with D2EHPA is $\mathrm{pH}$ dependent [10]. The $\mathrm{pH}$ of the leachate was varied in order to determine the effect of $\mathrm{pH}$ on the extraction of the metals out of the aqueous phase, and to determine the possibilities of achieving higher selectivity among the elements present. The organic phase used was 0.3 M D2EHPA in Solvent 70. The equilibrium $\mathrm{pH}$ after extraction with no modification was 1.1. The $\mathrm{pH}$ of the aqueous phase was adjusted by adding small amounts of 0.1 or $3 \mathrm{M} \mathrm{NaOH}$ to increase the $\mathrm{pH}$ or conc. $\mathrm{H}_{2} \mathrm{SO}_{4}$ to lower the $\mathrm{pH}$. A specific amount of the organic phase was added to the extraction system, which corresponded to the amount of the $\mathrm{NaOH}_{\mathrm{aq}}$ or conc. $\mathrm{H}_{2} \mathrm{SO}_{4}$ added. The equilibration was performed for $20 \mathrm{~min}$ by manual shaking before sampling to ensure re-equilibration. The sampled aqueous phases were diluted with $0.5 \mathrm{M}$ $\mathrm{HNO}_{3}$ and analyzed using ICP-OES.

\section{Stripping}

After determining the optimal extraction conditions, the metals needed to be stripped into the new aqueous phase for further reprocessing.

Stripping was performed by making the organic phase after extraction come into contact with $0.5,1,1.5,2,2.5$, and $3 \mathrm{M}$ hydrochloric acid $(\mathrm{HCl} ; 37 \%$, puriss, SigmaAldrich). The organic phase after the extraction was 
separated from the metal-depleted aqueous phase and made to come into contact with the stripping aqueous phase. A volume of $5 \mathrm{~mL}$ of each of the phases was added into a 20-mL vial and shaken manually for $20 \mathrm{~min}$ at a temperature of $25 \pm 1{ }^{\circ} \mathrm{C}$ and $\Theta=1$. After the stripping process, the stripping aqueous phases were sampled and diluted with $0.5 \mathrm{M} \mathrm{HNO}_{3}$ before carrying out analysis using ICP-OES.

\section{Results and Discussion}

\section{Determination of the Composition of the Leachate}

The measurable/detectable concentrations of $\mathrm{Nd}, \mathrm{Dy}, \mathrm{Pr}$, $\mathrm{Gd}, \mathrm{Co}$, and B in the leachate are shown in Table 1.

No Fe was detected with the measuring technique used. The $\mathrm{pH}$ value of the solution was measured using a MeterLab ${ }^{\mathrm{TM}}$ PHM $240 \mathrm{pH} /$ Ion Meter $\mathrm{pH}$ electrode and was determined to be 5.2. The concentration of the sulfate ion was kept constant throughout this research and was determined, after the precipitation of the sulfate ion using a $\mathrm{BaCl}_{2}$ solution, to be $\left[\mathrm{SO}_{4}{ }^{2-}\right]=23 \pm 1 \mathrm{mM}$.

\section{Effects of the Concentration of D2EHPA and the Diluent on Solvent Extraction}

Figure 1 shows the influence of D2EHPA concentrations on the extraction of $\mathrm{Nd}, \mathrm{Pr}, \mathrm{Gd}$, and Dy in various diluents.

The distribution ratios of the REEs increase with the increasing concentration of D2EHPA in all the diluents. The extractant clearly shows the higher distribution ratios for the HREEs (Dy and Gd) than the LREEs (Nd and Pr) in all the diluents. Similar trends were previously observed in a study by Mohammadi et al. [28]. The extraction efficiency is higher in the aliphatic diluents (hexane, octane, and Solvent 70), followed by toluene, and lowest in the polar diluents (cyclohexanone, 1-octanol, and chloroform).

Table 1 Concentrations of the metals of interest in the leachate measured using ICP-OES

\begin{tabular}{ll}
\hline Element & Concentration $(\mathrm{mM})$ \\
\hline $\mathrm{Nd}$ & $9.1 \pm 0.9$ \\
$\mathrm{Dy}$ & $2.7 \pm 0.6$ \\
$\mathrm{Pr}$ & $3.2 \pm 0.4$ \\
$\mathrm{Gd}$ & $0.69 \pm 0.16$ \\
$\mathrm{Co}$ & $0.17 \pm 0.09$ \\
$\mathrm{~B}$ & $0.55 \pm 0.14$ \\
Other $^{\mathrm{a}}$ & Below detection limit \\
\hline
\end{tabular}

The measurement was performed using ICP-OES after diluting the obtained leachate with $0.5 \mathrm{M} \mathrm{HNO}_{3}$

a Other constituent elements of the neodymium magnets like $\mathrm{Fe}, \mathrm{Ni}$, $\mathrm{Al}$ etc.
Cobalt was not extracted under any of the investigated conditions, and the distribution ratios remained around 0 . The distribution ratios for $\mathrm{B}$ were low at all concentrations and diluents. B was extracted up to $10 \%$ in cyclohexanone and 1-octanol. This can be attributed to the presence of carbonyl group in cyclohexanone and the hydroxyl group in 1-octanol, which could allow the diluent molecules to form complexes with $\mathrm{B}$ that are soluble in the organic phase. It was observed that $100 \%$ of all the REEs were transferred to the organic phase at 0.9 and 1.2 M D2EHPA in hexane and octane, while no Co or B was extracted.

As previously mentioned, the distribution ratios, and thus the extraction, are higher for the HREEs than those for the LREEs, which correspond to the decreasing ionic radii of these elements. The ionic radii of hydrolyzed $\operatorname{Pr}^{3+}$, $\mathrm{Nd}^{3+}, \mathrm{Gd}^{3+}$, and $\mathrm{Dy}^{3+}$ are accordingly 0.99, 0.983, 0.938, and $0.912 \AA$, respectively [29]. The outcome will correspondingly be the increased charge density with the decreasing ion radius. The smaller ion can contribute to the $\mathrm{REE}^{3+}$ binding to the D2EHPA molecule, governed by a cation-exchange mechanism, favoring the extraction of the HREEs in preference over the LREEs. According to the HSAB theory, which divides the acids and bases into hard and soft [30], $\mathrm{Co}^{2+}$ is a borderline acid, meaning it can act as a soft or hard acid, and it is difficult to predict its behavior since it can be influenced by various factors, like removal of hydrate water, steric effects, etc. In this case, $\mathrm{Co}^{2+}$ acts as a soft acid that did not form a complex with the D2EHPA from the organic phase.

To obtain a clearer picture of how diluents affect the extraction of different REEs, a graph was drawn to show the distribution ratios of $\mathrm{Nd}$ in $0.9 \mathrm{M}$ D2EHPA versus the dielectric constants $(\varepsilon)$ of the diluent (Fig. 2).

The distribution ratios of $\mathrm{Nd}$ in $0.9 \mathrm{M}$ D2EHPA diluted in various solvents are summarized in Fig. 2. The distribution ratios of $\mathrm{Nd}$ show a regular trend, decreasing with the polarity of the diluent in the following order: Solvent $70>$ octane $>$ hexane $>$ toluene $>$ cyclohexanone $>1$ octanol $>$ chloroform. The distribution ratios are one order higher in the aliphatic nonpolar diluents (Solvent 70, hexane, and octane) than in the aromatic and polar ones (toluene, cyclohexanone, 1-octanol, and chloroform). The fact that D2EHPA is a relatively nonpolar molecule, due to its 2-ethylhexyl chains, could explain the good solubility of the D2EHPA extractant in the aliphatic nonpolar diluents, thus leading to less aggregation of the extractant molecules and consequently resulting in higher distribution ratios for $\mathrm{Nd}$. This explanation does not apply for D2EHPA diluted with cyclohexanone, which gives distribution ratios for $\mathrm{Nd}$ higher than other polar diluents (1-octanol and chloroform) even though it has the largest dielectric constant (18.3). Nonetheless, this extraction-enhancing 

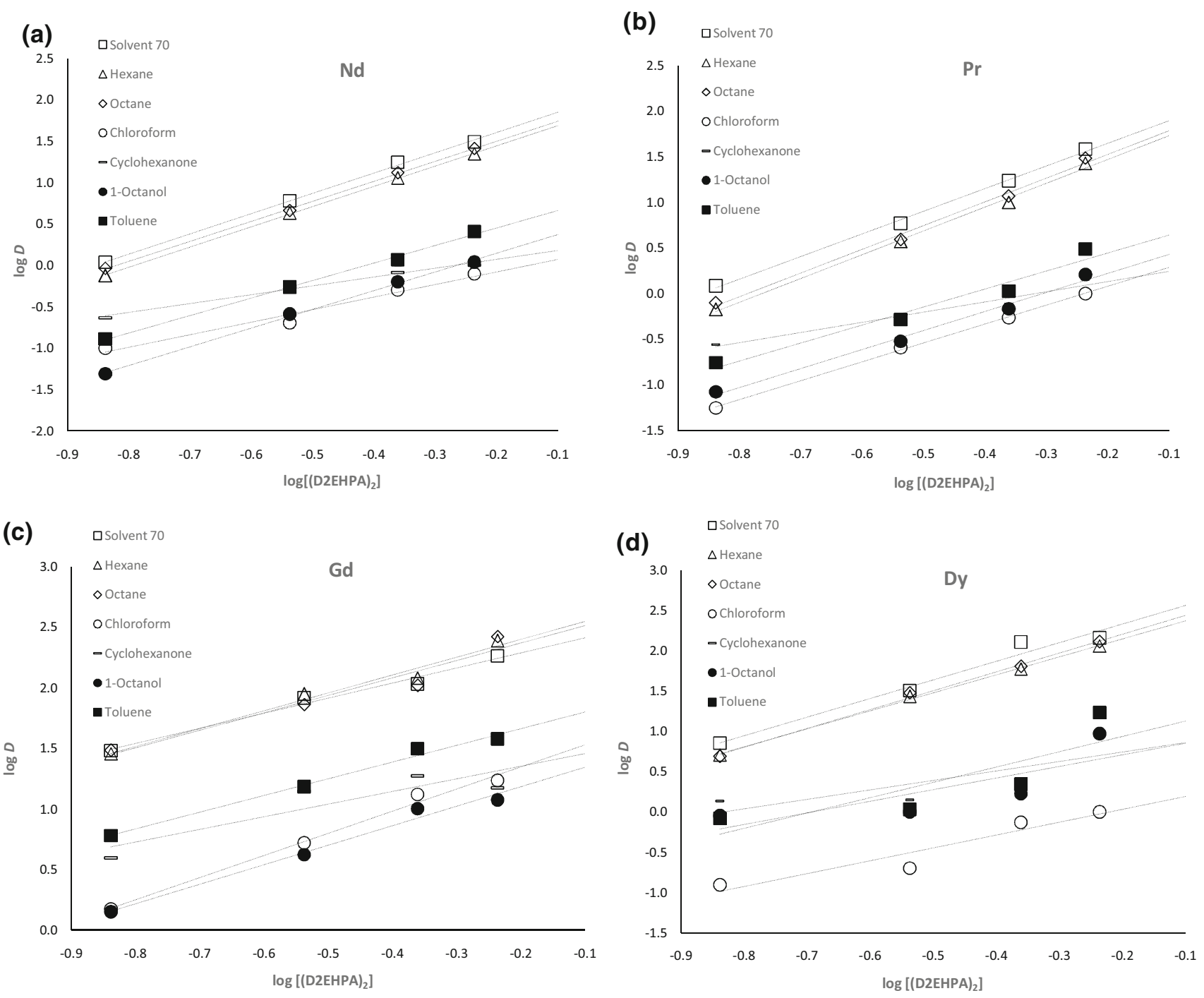

Fig. 1 The influences of D2EHPA concentrations on the extractions of $\mathbf{a} \mathrm{Nd}, \mathbf{b} \mathrm{Pr}, \mathbf{c} \mathrm{Gd}$, and $\mathbf{d} \mathrm{Dy}$ from the aqueous phase consisting of $9.11 \mathrm{~mm} \mathrm{Nd}, 2.71 \mathrm{mM}$ Dy, $3.16 \mathrm{~mm}$ Pr, $0.69 \mathrm{mM} \mathrm{Gd}, 0.17 \mathrm{mM}$ Co, and $0.55 \mathrm{mM} \mathrm{B}$, using different concentrations of $0.3,0.6,0.9$, and

phenomenon was expected, since cyclohexanone is a molecule with a donor atom, oxygen, for hydrogen bonding, but no active hydrogen atoms. This could cause it to form complexes with the REE ions in the solution, which could then be transferred to the organic phase [31]. This phenomenon could also explain the extraction of boron into the organic phase.

\section{Investigation of pH Effect on Extraction}

As expected, an increase of $\mathrm{pH}$ (lower proton concentration) led to higher metal extraction. This is a typical behavior for acidic extractants such as D2EHPA, and it can also be seen in Eq. 3 that lower $\mathrm{pH}$ values will shift the equilibrium to the left. On the other hand, the increase in proton concentration

1.2 M D2EHPA, respectively, in Solvent 70, hexane, octane, toluene, cyclohexanone, chloroform, and 1-octanol. The temperature was kept at $25 \pm 1{ }^{\circ} \mathrm{C}$, and the organic-to-aqueous phase ratio was $\Theta=1$. The error bars represent the standard deviation of a triplicate test

will favor the stripping reaction. It was observed that by increasing the $\mathrm{pH}$ values, Dy and Gd were extracted first, followed by $\mathrm{Nd}$ and $\mathrm{Pr}$. At around $0.1 \mathrm{M} \mathrm{H}^{+}$concentration, no extractions of $\mathrm{Co}$ and $\mathrm{B}$ whatsoever could be observed, while, when the $\mathrm{pH}$ value was increased to 1.9 , around $10 \%$ of B is extracted and around $20 \%$ of Co is extracted; hence, to avoid the co-extraction of the exogenes with the lanthanides as much as possible, the equilibrium $\mathrm{pH}$ should be kept at or below a value of 1 . The results also show that the light lanthanides ( $\mathrm{Nd}$ and $\mathrm{Pr}$ ) will be co-extracted with the heavy lanthanides in the first step of any method used with ratios of around 100\%:50\% (heavy:light). Increasing the equilibrium $\mathrm{pH}$ to 2 will lead to complete extraction of the lanthanides in the solution into the organic phase, but with the disadvantage of the increased co-extraction of $\mathrm{Co}$ and $\mathrm{B}$ in minor 


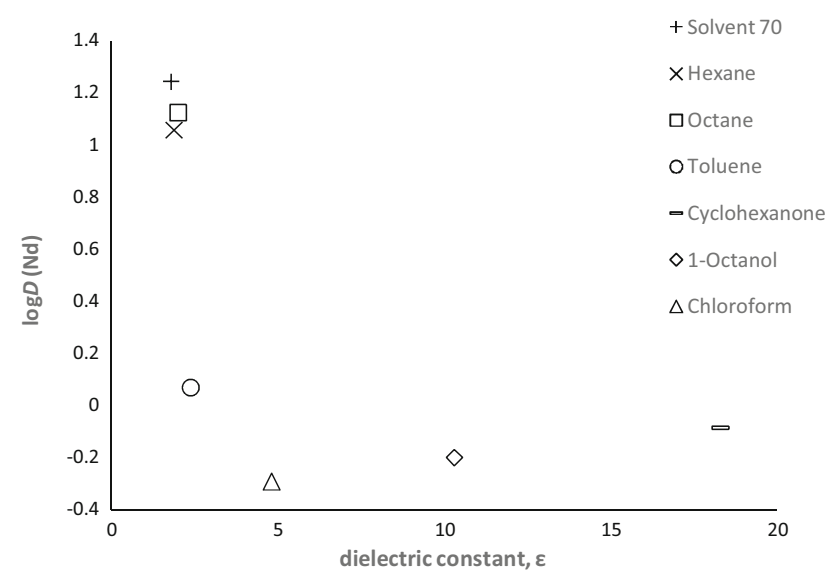

Fig. 2 Distribution ratios of $\mathrm{Nd}$ (logarithmic value) plotted as a function of the dielectric constants of the diluents. The organic phase used was 0.9 M D2EHPA diluted in Solvent 70, hexane, octane, toluene, cyclohexanone, chloroform, and 1-octanol. The aqueous phase consisted of $9.11 \mathrm{~mm} \mathrm{Nd}, 2.71 \mathrm{mM}$ Dy, $3.16 \mathrm{~mm}$ Pr, $0.69 \mathrm{mM}$ $\mathrm{Gd}, 0.17 \mathrm{mM} \mathrm{Co}$, and $0.55 \mathrm{mM} \mathrm{B}$. The extraction conditions were $25 \pm 1{ }^{\circ} \mathrm{C}$ and $\Theta=1$

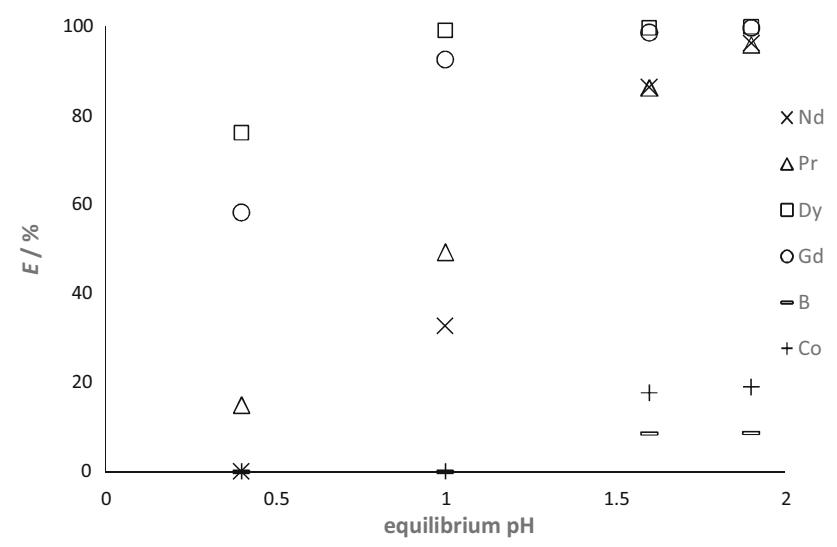

Fig. 3 Percentages of extraction of Nd, Pr, Dy, Gd, Co, and B from the neodymium magnet leachate consisting of $9.11 \mathrm{~mm} \mathrm{Nd}, 2.71 \mathrm{mM}$ Dy, $3.16 \mathrm{~mm}$ Pr, $0.69 \mathrm{mM} \mathrm{Gd}, 0.17 \mathrm{mM} \mathrm{Co}$, and $0.55 \mathrm{mM}$ B with varied equilibrium $\mathrm{pH}$ values. The extraction conditions were $25 \pm 1{ }^{\circ} \mathrm{C}$ and $\Theta=1$. The organic phase used was 0.3 M D2EHPA in solvent 70 quantities. This could lead to more extraction stages in, for example, a mixer-settler (Fig. 3).

\section{Separation Factors Between the HREEs and the LREEs}

To further investigate the selectivity between the LREEs and the HREEs of these extractions, the separation factors were calculated. The calculated separation factors are shown numerically in Table 2 .

The highest separation factors between Dy and Nd were achieved at $0.3 \%$ D2EHPA in hexane. Under these conditions, around $50 \%$ of $\mathrm{Nd}$ and $\mathrm{Pr}$ were extracted, and around $99 \%$ of Dy and Gd extractions were realized. The $\alpha \mathrm{Dy} /(\mathrm{Nd} \&$ Pr) kept decreasing with the increasing concentration of D2EHPA for the Solvent 70, while it remained below 10 for other concentrations of D2EHPA for all the other diluents. As for $\mathrm{Gd} / \mathrm{Nd}$ and $\mathrm{Gd} / \mathrm{Pr}$, the best separation factor was also found to be in $0.3 \mathrm{M}$ hexane. It can also be noted that the separation factors between Dy and other LREEs are one order lower than those of Gd and other LREEs. Even though it is expected that Dy and Gd should have similar distribution ratios, as seen from Table 1, the concentration of Dy is four times higher than that of Gd in the leachate. This concentration difference might have led to the much higher distribution ratios for Gd than those for Dy, leading consequently to these results. Since Dy is present in a much higher concentration and its current price is around 10 times higher than that of $\mathrm{Gd}$, the organic phase consisting of $0.3 \mathrm{M}$ D2EHPA diluted in hexane was chosen as the best organic phase for the separations of Dy and Gd from the other LREEs, especially considering the small amounts of B and Co being extracted. Future work on the scale-up process in a mixer-settler is proposed.

\section{Stripping}

The stripping experiments were conducted for the aqueous phases after extraction with 0.3 M D2EHPA in hexane and

Table 2 Separation factors between the HREEs and the LREEs after extraction

\begin{tabular}{|c|c|c|c|c|c|}
\hline & [D2EHPA]/M & $\alpha_{\mathrm{Dy} / \mathrm{Nd}}$ & $\alpha_{\mathrm{Dy} / \operatorname{Pr}}$ & $\alpha_{\mathrm{Gd} / \mathrm{Nd}}$ & $\alpha_{\mathrm{Gd} / \mathrm{Pr}}$ \\
\hline \multirow[t]{4}{*}{ Solvent 70} & 0.3 & $6.55 \pm 3.2$ & $5.85 \pm 3.1$ & $27.7 \pm 1.2$ & $24.8 \pm 0.9$ \\
\hline & 0.6 & $5.34 \pm 0.7$ & $5.43 \pm 0.5$ & $13.7 \pm 0.2$ & $14.0 \pm 0.3$ \\
\hline & 0.9 & $7.3 \pm 0.1$ & $7.4 \pm 0.2$ & $6.1 \pm 0.6$ & $6.2 \pm 0.2$ \\
\hline & 1.2 & $4.6 \pm 0.4$ & $3.8 \pm 0.5$ & $5.8 \pm 0.5$ & $4.7 \pm 0.4$ \\
\hline \multirow[t]{4}{*}{ Hexane } & 0.3 & $6.8 \pm 0.6$ & $7.6 \pm 0.8$ & $37.7 \pm 1.2$ & $42.4 \pm 1.6$ \\
\hline & 0.6 & $6.3 \pm 0.7$ & $7.27 \pm 0.4$ & $20.6 \pm 0.9$ & $23.7 \pm 1.2$ \\
\hline & 0.9 & $5.3 \pm 0.6$ & $5.94 \pm 0.3$ & $10.5 \pm 0.7$ & $11.9 \pm 0.5$ \\
\hline & 1.2 & $5.1 \pm 0.3$ & $4.28 \pm 0.6$ & $10.9 \pm 0.5$ & $9.2 \pm 0.4$ \\
\hline Octane & 0.3 & $5.8 \pm 0.2$ & $6.1 \pm 0.4$ & $33.1 \pm 1.2$ & $38.3 \pm 0.9$ \\
\hline
\end{tabular}


Table 2 continued

\begin{tabular}{|c|c|c|c|c|c|}
\hline & $\begin{array}{l}{[\mathrm{D} 2 \mathrm{EHPA}] / \mathrm{M}} \\
0.6\end{array}$ & $\begin{array}{l}\alpha_{\text {Dy/Nd }} \\
6.5 \pm 0.4\end{array}$ & $\begin{array}{l}\alpha_{\mathrm{Dy} / \mathrm{Pr}} \\
7.6 \pm 0.3\end{array}$ & $\begin{array}{l}\alpha_{\mathrm{Gd} / \mathrm{Nd}} \\
15.7 \pm 0.9\end{array}$ & $\begin{array}{l}\alpha_{\mathrm{Gd} / \mathrm{Pr}} \\
18.5 \pm 0.7\end{array}$ \\
\hline & 0.9 & $4.8 \pm 0.3$ & $5.4 \pm 0.7$ & $7.9 \pm 0.6$ & $8.9 \pm 0.5$ \\
\hline & 1.2 & $5.0 \pm 0.3$ & $4.2 \pm 0.6$ & $10.2 \pm 0.9$ & $8.6 \pm 0.4$ \\
\hline \multirow[t]{4}{*}{ Toluene } & 0.3 & $6.5 \pm 0.7$ & $4.8 \pm 0.9$ & $36.8 \pm 0.9$ & $34.5 \pm 0.7$ \\
\hline & 0.6 & $2.0 \pm 0.4$ & $2.1 \pm 0.1$ & $27.9 \pm 0.6$ & $29.3 \pm 0.5$ \\
\hline & 0.9 & $1.9 \pm 0.3$ & $2.1 \pm 0.3$ & $26.7 \pm 0.9$ & $29.5 \pm 0.5$ \\
\hline & 1.2 & $6.7 \pm 0.4$ & $5.5 \pm 0.8$ & $14.8 \pm 0.6$ & $12.2 \pm 0.7$ \\
\hline \multirow[t]{4}{*}{ Cyclohexanone } & 0.3 & $5.9 \pm 0.3$ & $4.9 \pm 0.3$ & $17.0 \pm 0.9$ & $14.3 \pm 0.7$ \\
\hline & 0.6 & $2.5 \pm 0.4$ & $2.6 \pm 0.1$ & $25.1 \pm 0.7$ & $25.8 \pm 0.2$ \\
\hline & 0.9 & $2.7 \pm 0.2$ & $3.0 \pm 0.3$ & $22.7 \pm 0.2$ & $24.4 \pm 0.4$ \\
\hline & 1.2 & $9.0 \pm 0.6$ & $6.3 \pm 0.5$ & $14.8 \pm 1.2$ & $10.4 \pm 0.9$ \\
\hline \multirow[t]{4}{*}{ Chloroform } & 0.3 & $5.2 \pm 0.5$ & $6.0 \pm 0.1$ & $25.0 \pm 0.2$ & $26.8 \pm 0.4$ \\
\hline & 0.6 & $3.5 \pm 0.4$ & $2.7 \pm 0.5$ & $26.2 \pm 0.9$ & $20.5 \pm 0.4$ \\
\hline & 0.9 & $1.5 \pm 0.1$ & $1.4 \pm 0.3$ & $26.1 \pm 0.6$ & $24.1 \pm 0.5$ \\
\hline & 1.2 & $0.9 \pm 0.3$ & $0.9 \pm 0.1$ & $17.0 \pm 0.3$ & $17.2 \pm 0.3$ \\
\hline \multirow[t]{4}{*}{ 1-Octanol } & 0.3 & $4.9 \pm 0.6$ & $5.1 \pm 0.8$ & $29.0 \pm 1.2$ & $26.9 \pm 0.8$ \\
\hline & 0.6 & $3.9 \pm 0.4$ & $3.3 \pm 0.1$ & $16.3 \pm 0.7$ & $14.0 \pm 0.9$ \\
\hline & 0.9 & $2.7 \pm 0.3$ & $2.5 \pm 0.6$ & $15.9 \pm 0.5$ & $14.8 \pm 0.8$ \\
\hline & 1.2 & $8.5 \pm 0.9$ & $5.8 \pm 0.8$ & $10.9 \pm 1.0$ & $7.34 \pm 0.9$ \\
\hline
\end{tabular}

The concentrations of D2EHPA were $0.3,0.6,0.9$, and $1.2 \mathrm{~mol} / \mathrm{L}$ in Solvent 70, hexane, octane, toluene, cyclohexanone, chloroform, and 1-octanol. The aqueous phase consisted of $9.11 \mathrm{~mm} \mathrm{Nd}, 2.71 \mathrm{mM}$ Dy, $3.16 \mathrm{mM}$ Pr, $0.69 \mathrm{mM} \mathrm{Gd}, 0.17 \mathrm{mM} \mathrm{Co}$, and $0.55 \mathrm{mM}$ B. The temperature was kept at $25 \pm 1{ }^{\circ} \mathrm{C}$ and the organic-to-aqueous phase ratio was $\Theta=1$

1.2 M D2EHPA in octane. At 0.3 M D2EHPA in hexane, the highest separation factors between the HREEs and the LREEs were achieved, and at $1.2 \mathrm{M}$ D2EHPA, the REEs were completely extracted out of the leachate as a group, leaving the exogenous metals $(\mathrm{Co}, \mathrm{B})$ in the solution; so these two cases were found to be the most interesting for future process developments, if needed. The elements were stripped back into the new aqueous phase using hydrochloric acid at the concentrations of $0.5,1,1.5,2,2.5$, and $3 \mathrm{M}$ for $5 \mathrm{~min}$. The organic phase after extraction was made to come into contact with hydrochloric acid at the aforementioned concentrations and shaken using an Ika Vibrax Vxr shaking machine at a temperature of $25 \pm 1{ }^{\circ} \mathrm{C}$ with an $\mathrm{O}: \mathrm{A}=1: 1$. The results of the stripping are shown in Fig. 4.

The metals are stripped at $100 \%$ efficiency at $2 \mathrm{M} \mathrm{HCl}$ or higher, which is in accordance with Eq. 3. This means that increasing the concentration of $\mathrm{H}^{+}$ions in the aqueous solution will shift the reaction to the left.

\section{Conclusions}

This work was focused on the extractions of the HREEs and the LREEs from a real leachate acquired from a neodymium magnet leachate in sulfuric acid media, and to find the most suitable separation conditions between these. The general extraction order is HREEs > LREEs, which was expected. The distribution ratios, and consequently the extraction efficiencies, increased as the D2EHPA concentration increased and the acidity decreased. The kinetics of the extraction were monitored for $0.6 \mathrm{M}$ D2EHPA in Solvent 70, hexane, octane, chloroform, cyclohexanone, 1-octanol, and toluene. It was shown that the equilibrium is reached within 3 min for all diluents except for cyclohexanone and 1-octanol, in which cases, the equilibrium is reached within $5 \mathrm{~min}$.

It was concluded that the most suitable diluents for the solvent extraction of REEs with D2EHPA (where aliphatic diluents were used) were hexane, octane, and Solvent 70 . The slightly polar diluents, i.e., cyclohexanone, chloroform, and 1-octanol, showed the lowest distribution ratios for the extracted REEs. Toluene was found to be somewhere between the two groups previously mentioned, according to the obtained distribution ratios for the REEs.

It has also been demonstrated that at $0.9 \mathrm{M}$ and $1.2 \mathrm{M}$ D2EHPA concentrations in hexane and octane, the REEs are completely extracted out of the leachate solution as a group, leaving the exogenes (Co and $\mathrm{B}$ ) within the solution.

Concerning the separation between the HREEs and the LREEs, the best separation factors were obtained when extracting with $0.3 \mathrm{M}$ D2EHPA in hexane. Under such conditions, almost all the Dy and Gd were extracted from 

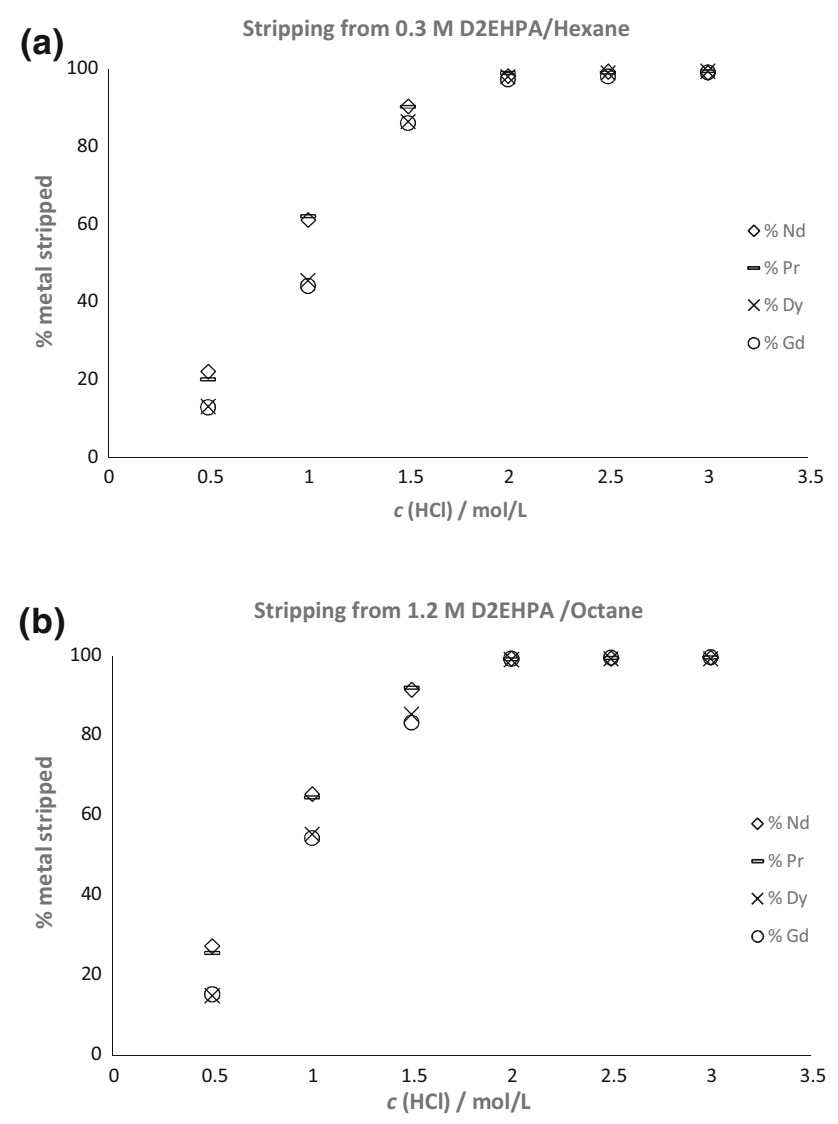

Fig. 4 The back stripping results of the REEs in the aqueous solution from a $0.3 \mathrm{M}$ D2EHPA in Solvent 70 and b 1.2 M D2EHPA in octane after extraction. The stripping agents used were aqueous solutions of $0.5,1,1.5,2,2.5$, and $3 \mathrm{M}$ hydrochloric acid, and the plotted values on the graph represent the initial values of the $\mathrm{HCl}$ concentration. The stripping conditions were a temperature of $25 \pm 1{ }^{\circ} \mathrm{C}$ and an organicto-aqueous phase ratio of $\Theta=1$. Shaking time was $20 \mathrm{~min}$

the solution, while around half of the amount (50\%) of the LREEs present were extracted.

The stripping studies were performed using the organic phases after the extraction with 0.3 M D2EHPA in hexane and 1.2 M D2EHPA in octane. It has been shown that the complete stripping of the elements back into the aqueous phase is achieved at $2 \mathrm{M} \mathrm{HCl}$ or higher.

These were, however, the observations made on a small scale $(3.5 \mathrm{~mL})$, and for further developing the process, larger amounts of the leachate are required, and mixersettler studies are recommended with 0.3 M D2EHPA in Solvent 70 and $1.2 \mathrm{M}$ of D2EHPA in octane to develop a large-scale process in the future.

Acknowledgements The present research has received funding from the European Community's Seventh Framework Programme ([FP7/ 2007-2013]) under Grant Agreement No. 607411 (MC-ITN EREAN: European Rare Earth Magnet Recycling Network). This article reflects only the views of the authors, exempting the Community from any liability. Project website: http://www.erean.eu.
Open Access This article is distributed under the terms of the Creative Commons Attribution 4.0 International License (http://crea tivecommons.org/licenses/by/4.0/), which permits unrestricted use, distribution, and reproduction in any medium, provided you give appropriate credit to the original author(s) and the source, provide a link to the Creative Commons license, and indicate if changes were made.

\section{References}

1. Swain B, Otu EO (2011) Competitive extraction of lanthanides by solvent extraction using Cyanex 272: analysis, classification and mechanism. Sep Purif Technol 83:82-90

2. Binnemans K, Jones PT, Blanpain B, Van Gerven T, Yang Y, Walton A, Buchert M (2013) Recycling of rare earths: a critical review. J Clean Prod 51:1-22

3. European Commission (2014) Critical raw materials for the EU in Report of the Ad hoc Working Group on defining critical raw materials. European Commission, Washington, DC

4. Polyakov EG, Sibilev AS (2015) Recycling rare-earth-metal wastes by pyrometallurgical methods. Metallurgist 59(5):368-373

5. Bandara HMD, Apelian JW, Emmert D, Marion H (2015) Rare earth recycling: forecast of recoverable $\mathrm{Nd}$ from shredder scrap and influence of recycling rates on price volatility. J Sustain Metall 1(3):179-188

6. Tunsu C, Petranikova M, Gergorić M, Ekberg C, Retegan T (2015) Reclaiming rare earth elements from end-of-life products: a review of the perspectives for urban mining using hydrometallurgical unit operations. Hydrometallurgy 156:239-258

7. Lyman JW, Palmer GR (1993) Recycling of neodymium iron boron magnet scrap. United States Department of the Interior, Washington, DC

8. Riano S, Binnemans K (2015) Extraction and separation of neodymium and dysprosium from used $\mathrm{NdFeB}$ magnets: an application of ionic liquids in solvent extraction towards the recycling of magnets. Green Chem 17(5):2931-2942

9. Swain B, Jeong J, Lee J, Lee G (2008) Development of process flow sheet for recovery of high pure cobalt from sulfate leach liquor of LIB industry waste: a mathematical model correlation to predict optimum operational conditions. Sep Purif Technol 63(2):360-369

10. Xie F, Zhang TA, Dreisinger D, Doyle F (2014) A critical review on solvent extraction of rare earths from aqueous solutions. Miner Eng 56:10-28

11. Kuipa PK, Hughes MA (2002) Diluent effect on the solvent extraction rate of copper. Sep Sci Technol 37:1135-1152

12. Anticó E, Masana A, Hidalgo M, Salvadó V, Iglesias M, Valiente M (1996) Solvent extraction of yttrium from chloride media by di(2-ethylhexyl)phosphoric acid in kerosene. Speciation studies and gel formation. Anal Chim Acta 327(3):267-276

13. Lee C, Chen Y, Liao C, Popuri SR, Tsai S, Hung C (2013) Selective leaching process for neodymium recovery from scrap Nd-Fe-B magnet. Metall Mater Trans 44(13):5825-5833

14. Yoon H-S et al (2014) Leaching kinetics of neodymium in sulfuric acid from E-scrap of NdFeB permanent magnet. Korean J Chem Eng 31(4):706-711

15. Önal MAR, Borra CR, Guo M, Blanpain B, Van Gerven T (2015) Recycling of NdFeB magnets using sulfation, selective roasting, and water leaching. J Sustain Metall 1(3):199-215

16. Nash KL, Jensen MP (2001) Analytical-scale separations of the lanthanides: a review of techniques and fundamentals. Sep Sci Technol 36(5-6):1257-1282 
17. Cotton S (2006) Lanthanide and actinide chemistry. Wiley, New York

18. Kim JS, Kumar BN, Radhika S, Kantan ML, Reddy BR (2012) Studies on selection of solvent extractant system for the separation of trivalent $\mathrm{Sm}, \mathrm{Gd}$, Dy and Y from chloride solutions. Int $\mathbf{J}$ Miner Process 112-113:37-42

19. Henderson P (1984) Rare earth element geochemistry. Elsevier, Amsterdam

20. Kraikaew J, Srinuttrakul W (2005) Solvent extraction study of rare earths from nitrate medium by the mixtures of TBP and D2EHPA in kerosene. J Met Mater Miner 15:89-95

21. Peppard DW, Mason GW (1961) Liquid-liquid extraction of trivalent rare earths using acidic phosphonates as extractants. Macmillan, New York, pp 37-50

22. Schiff NN (1968) Molybdenum corporation of America's mountain pass europium process. In: AIME annual meeting, New York

23. Nash KL, Chris GR (1997) Separation of elements. Springer, Oxford

24. Yang J (2015) Process development for extraction and separation of In and Y from discarded flat panel displays. Chalmers University of Technology, Göteborg
25. Niinae M (1991) Effect of diluents on extraction of cobalt with D2EHPA. J Jpn Inst Met Mater 55(3):310-315

26. Basualto C, Valenzuela F, Molina L, Munoz JP, Fuentesand E, Sapag J (2013) Study of the solvent extraction of the lighter lanthanide metal ions by means of organophosphorus extractants. J Chil Chem Soc 58(2):1785-1789

27. Yang J, Ekberg C (2014) Optimization of indium recovery and separation from LCD waste by solvent extraction with bis(2ethylhexyl) phosphate (D2EHPA). Int J Chem Eng 2014:9

28. Mohammadi M, Forsberg K, Kloo L, Martinez De La Cruz J, Rasmuson $\AA$ (2015) Separation of ND(III), DY(III) and Y(III) by solvent extraction using D2EHPA and EHEHPA. Hydrometallurgy 156:215-224

29. Cotton S (2006) Lanthanide and actinide chemistry. Wiley, Chichester

30. Rydberg J, Cox M, Musicas C, Choppin GR (2004) Solvent extraction principles and practice. Taylor \& Francis, Boca Raton

31. Yang J, Ekberg C (2013) Indium recovery from discarded LCD panel glass by solvent extraction. Hydrometallurgy 137:68-77 